Article

\title{
Formal Binary Logic, Tidal Forces and the Equivalence Principle
}

\author{
Holger Döring
}

IQ-Wissen, Germany

* Correspondence: haw-doering@t-online.de

\begin{abstract}
Mass plays a strange multiple role in classical physics. This results in a difference between gravity and all other forces - a difference that stood at the beginning of Einstein's development of his general theory of relativity. The equivalence principle (EP) deals with homogenious gravitational fields, which, in fact, don't exist in nature. There is only an approximation for this field in Einstein-Lift possible for local situations. Nevertheless this EP can be true, because in classical formal binary logic there can be formulated a true conclusion from a wrong premise or assumption.
\end{abstract}

Key-words: equivalence-principle; Einstein-Lift; EP; formal binary logic; inhomogenious gravityfield;acceleration- field; radial-field;tidal-forces

\section{Introduction}

In 1905, Albert Einstein created a new basis for the physical laws of physics with his special theory of relativity. However, one area of physics was absolutely not compatible with the new ideas of space and time: gravitational force, described by Newton's law of gravitation. The special theory of relativity only seemed to create the framework for gravity-free physics. It was only years later that Einstein was able to classify gravitation into a new picture of space and time with a completely new theory, the general theory of relativity. The first step on the way to this new theory was the realization that even in a gravitational field there are reference systems in which the rules of gravity-free physics apply, and thus the physics of the special theory of relativity - at least approximately and as long as the focus ywhich is considered on one limit to a small segment of space and time. This circumstance results from the equivalence principle formulated by Einstein, and this in turn is related to special properties of free fall.[7.]

Einstein came to believe that this inability to distinguish applies not only to observations of falling bodies but to any physical measurements. With no experiment, based on no physical law, he postulated, one can determine whether one is in gravitation-free space or in a falling cabin in the gravitational field. This is the content of the so-called equivalence principle. In other words, this means: The same physical laws apply in every freefalling reference system as they also apply in gravitation-free physics, i.e. in the physics of the special theory of relativity. This form of the equivalence principle is also often called Einstein's equivalence principle, in contrast to the so-called weak equivalence principle, which states that all bodies that are in the same place in the gravitational field fall at the same speed.[8.],[9.].

Einstein introduced his lift-example to show the equivalence between a constant accelerating field with homogenious, parallel field-lines and a gravitational field under the same conditions. In fact, there are in reality no homogenious gravity-fields in nature, only radial-fields.The approximation of Einstein is usually used in local space for showing the equivalence of inertial mass and gravity mass.[3.],[4.]

Suppose the passenger is in a closed booth, cut off from the outside world. Bodies he drops fall to the ground at an accelerated rate, just as he is used to from Earth. Can he conclude from this that he and his cabin are actually in a gravitational field like that of Earth, as outlined in the following figure 1 ? 
The formal thinking is:

No, because everything could be completely different. Theoretically, you could also be in space, far removed from any major mass accumulations and their gravitational influence. That is when your cabin is on board a rocket that is being accelerated by its engine at a rate of 9.81 meters per second squared.[11.]. But this suppose is false.

In such a situation, the cabin floor would accelerate toward any object he dropswith exactly the same acceleration as gravity causes objects to fall to the ground here on Earth. From his point of view has an observer in the cabin, the two situations - gravity causes objects to fall to the ground at [4.]an accelerated rate, the cabin floor accelerates toward objects - are very distinguishable.

Strictly speaking, the statements made so far about the equivalence of gravitation and acceleration only apply in an absolutely homogeneous gravitational field. In such a gravitational field, all freely falling bodies are actually accelerated in the same way, i.e. in the same direction and with the same force. In fact, a researcher inside a locked cabin cannot distinguish between this gravitational acceleration and the acceleration from, say, a rocket engine. However, real gravitational fields are always inhomogeneous in some way. Let's take the gravitational effect of the earth. Here on the surface of the earth, which is huge compared to us, we are used to it: bodies fall to the ground, and if we disregard effects such as air friction, this always happens with the same acceleration and always in the same direction, i.e. "down". But if we take a closer look, that's not entirely true. This becomes clear when we consider, for example, the following gigantic elevator, which falls towards the earth.

In fact, the premise is wrong, because there are no homogenious gravity-fields. Nevertheless, the conclusion of EP can be true, because there can be a right statement from a wrong assumption after the rules of classical aristotelic formal binary-logic.

\section{Calculation}

Let there be an Einstein-Lift with lenghth of ten meters and heigth of ten meters.It may be in free fall of one hundred kilometers above earth-surface or it may be pulled above by an acceleration-field of a rocket-machine or long rope above.There are no windows inside, so the lift-passenger has no possibilty to decide, if he is in a free fall over a planet surface or accelerated by a rocket machine.He does not know, that he is in $100 \mathrm{~km}$ height above earth.

But what about the field lines?

If there is an acceleration-field, the passenger assumes a lot of homogenious fieldlines, if there is a planet surface nearby (under him) with a gravity field, this field has to be radial, not homogenious, so there will be no parallel field-lines but there are adding differences with adding heigths over planetary center (see Figure 1).This is called tidalforce.There are always tidal-forces in gravity fields and they can't be neglected. So he can try to calculate a deviation from parallelity. 


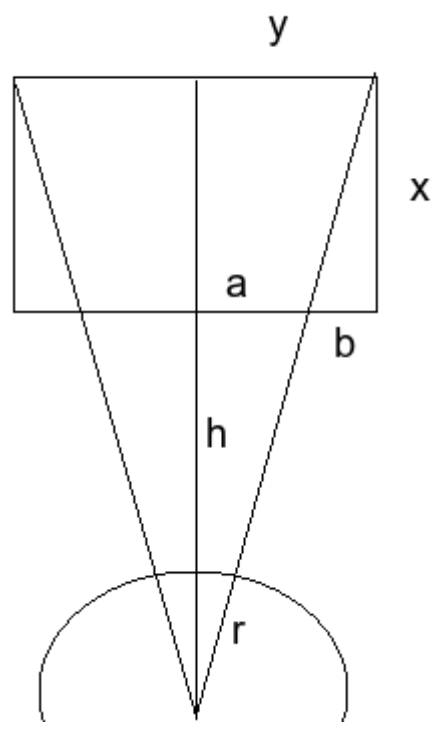

Figure 1. Einstein-Lift above earth with three radial gravity field-lines in a heigth of $h=$ $100 \mathrm{~km}$. (No scaling but only qualitative drawings.)

Here is seen that the cabin and some probe masses in it,don't fall parallel to each other, but towards one and the same point, the center of the earth. For the observer in the falling cabin, this is expressed in the fact that the probes move very slightly towards each other. This is what is known as a tidal effect. On closer observation, a freely falling observer can also determine from such effects that he is in an (natural inhomogeneous) gravitational field and not in gravitation-free space. To put it more precisely, the equivalence principle reads: The same physical laws apply in every freely falling reference system as in the special theory of relativity as long as tidal effects can be neglected.But they can't, because in every inhomogenious, natural, real gravitational field there are tidal forces!

So far, so good, but also: so simplified. Strictly speaking, the statements made so far about the equivalence of gravitation and acceleration only apply in an absolutely homogeneous gravitational field. In such a gravitational field, all freely falling bodies are actually accelerated in the same way, i.e. in the same direction and with the same force. In fact, a researcher inside a locked cabin cannot distinguish between this gravitational acceleration and the acceleration from, say, a rocket engine. However, real gravitational fields are always inhomogeneous in some way.

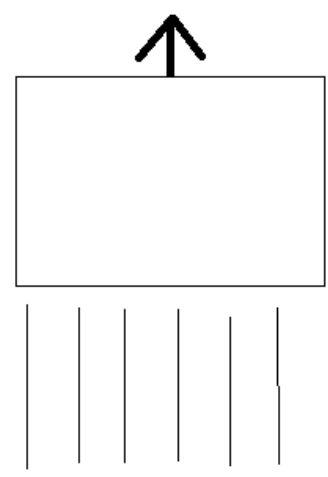

Figure 2. Einstein-Lift in a homogenious acceleration-field with Force upwards.

How can the researcher measure now his actual situation?

Now the passenger installs two lasers in the middle of the top deck of the lift at the edges, which beams shows downwards.On the floor he installs a line of photometers in 
the middle line of the lift, to register the light beams.The light beams will follow the field lines of the outer-field. If he measures a deviation from parallellity, there is a planetary radial-field down of his lift .If there is no such deviation, the lift is accelerating with a homogenious a-field from a rocket-machine or a rope.

He takes a calibration of his measuring in the fact, that a difference of, say ten Bohrradii $\left(r_{B}=5,29 * 10^{-} 11 \mathrm{~m}\right)$ from parallelity between the upper deck and the ground of the lift would be a significant sign for measuring a radial gravity field.

It is the fact that basically measuring processes in the lift are possible and there is supposed that the passenger can have a useful measuring-instrument which he can calibrate accurately to do his measuring processes he want.But he has no windows.

If he measures a deviation of $b=7,718 * 10^{-6} \mathrm{~m}$, he can deduce by a short calculation the size of $h+$ rabove his planet. (See calculation below).If he assumes, that the radius is from planet Earth $r=6378 \mathrm{~km}$, he can calculate the heigth to $h=100 \mathrm{~km}$ with proportional lengths at same angles. (This makes b equal to 145905 Bohr-radii, a very great distance). If there is no deviation of the light beams from parallellity in his measuring limit of ten Bohr-radii he is inside of an accelerating lift with a rocket machine or a rope. So he can make a difference between the two fields. There is no equality between this two fields, only a small deviation of tidal-forces but this leads to the conclusion, that there is no exact correspondence between a gravity field and an accelerating field but that this only a (possibly good) approximation for local states, where the curving of the field is weak. But how can then physically be deduced, that inertia mass is equal to gravity mass? Is this only an approximation, too? No, because in Einstein's theory of gravitation, the general theory of relativity, the reaction of bodies to gravitational effects is explained purely geometrically - masses distort the geometry of space and time, and all bodies follow the straightest possible paths in this distorted space-time. The artificial dichotomy that follows from the concept of force - the force dependent on the gravitational mass on the one hand, and the reaction to it dependent on the inertial mass on the other - is replaced by a law in which the equal treatment of all bodies is built in at the lowest level: that all bodies experience the same gravitational acceleration in a given situation is because their motion is directly governed by the properties of the space-time around them.

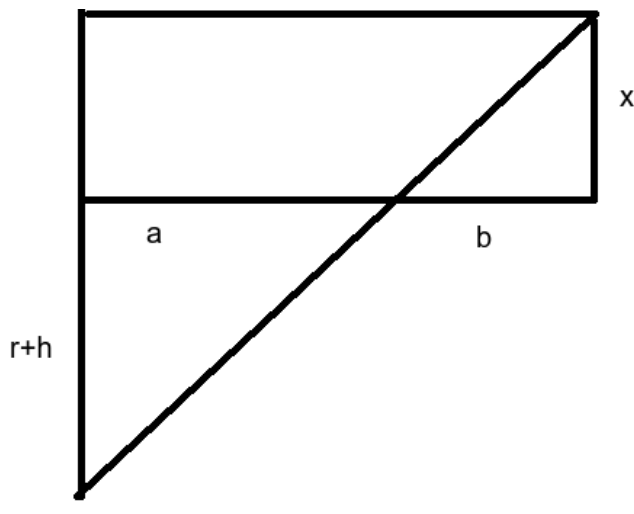

Figure 3. The geometric connection between different lift-sizes to calculate length a resp. b by proportional lengths at the same angle.

The calculation is:

$\frac{a}{h+r}=\frac{a+b}{h+r+x}$

Data:

$$
h=100 \mathrm{~km} ; r=6378 \mathrm{~km} ; x=10 \mathrm{~m} ; a+b=5 \mathrm{~m}
$$

This short calculation leads to 


$$
a=4,999992282 m ; b=7.71841971 * 10^{-6} m
$$

This value of $b$ is a great deviation of parallelity (at any rate some micrometer), which measuring with light beams must be easy.There is no bending of the light-beams in gravity-field, caused by GRT, because there are neither tangential lines nor is there a propagating of the beams in an angle against gravity-fieldlines but they follow these lines. (Of course, there is energy-loss resp. frequency changing caused by gravty field explained by GRT but that fact doensn't belong to our problem).If the passenger is measuring this value, he can calculate his heigth over planet-surface, under the condition, that he knows the radius of the planet.

$$
h=\frac{a \cdot x}{b}-r
$$

The value $x$ is the heigth of the lift, and $b$ are measured resp. calculated and $r$ the passenger has to know from outer sources.

\section{Remark:}

In fact, one can state more specifically how tidal effects can be kept small. On the one hand, by limiting your observations to a very small region of space. If you drop two bodies a few meters apart on the ground here, you will hardly be able to prove the effect. Secondly, the observation period plays a role. If you only watch the balls in the animation for a very short period of time, you will have great difficulty in noticing that they are approaching each other. Therefore, in addition to the equivalence principle, there is an exact formulation as a useful guide that says: In infinitely small ("infinitesimal") space-time regions, a reference system can always be found - an infinitely small free-falling cabin, viewed over an infinitely short observation period - in which the same physical laws apply as in the special theory of relativity. If the cabin is small enough and the observation period is short enough, the deviation of the physical laws inside the cabin from those of the special theory of relativity can be kept as small as you like. Is this so? No.

What now about the introduction of "infinitesimal inertial systems" to clear this phenomenon of approximation? There is to say, that this concept is pure nonsense because there is to make physics not mathematics (example given: see the discussion between Richard Feynman and the mathematicians about the theorem of Banach-Tarski[12.]).The smallest real length is the planck-length and an inertial system has to have at least the seize to describe the (constant) moving of a real particle like an electron, proton or neutrino etc. So the real minimal seize of an existing inertial system in reality is determined by this particle diameters and this is not „infinitesimal“. The size of the IS should be at least of double (or three times) diameter of the particle in every axe to describe a real movement of this particle. (Of course movements of less then a diameter can be described also but are in these connections here not defined as real "movement".).

Answer: in a real inertial system of at least minimal seize there are tidal-forces, if there is assumed, that this IS is in free-fall in a gravity field. This deviation from parallel field-lines can be calculated in principle, dependend only from the measuring tools.

\section{Conclusion}

There is no exact correspondence between a real radial gravity field and an acceleration field with constant value and parallel field lines. Nevertheless Einstein derived from this approximation the equivalence of inertia mass and gravity mass (heavy mass).Although his premisse is wrong, it may be true, that his conclusion is accurate.

It is called a false premise when a conclusion is drawn based on a false or unknown statement (the premise). It is important to note that an argument can contain both a false premise and a false conclusion but the conclusion may be true, either. 
In fact, there is no empirical or logical pillar for the EP. But it may be true or not.

\section{Summary:}

There are three possibilities for solutions of this problem:

First: Einsteins EP doesn't hold.

Second: The premise is wrong but the formal logic is right and the EP is real.

Third: there are no homogenious accelerating fields in nature.

But:

Test particles, regardless of their composition or other nature, follow the same fall curve if their initial location and velocity match. In the Newtonian sense, inert and passive heavy masses, between which one cannot distinguish in GRT, are equivalent. The fact that all test particles go through the same fall curves in GRT is due to the fact that the Lagrangian of GRT does not change when the coordinates change. The equivalence principle is thus present as a fundamental symmetry in the theory.[10.]

The observation of a violation of the principle of equivalence would therefore show that the GRT would only have limited validity. With today's measurement accuracy, no deviations from the equivalence principle have been observed.[2.],[6.].

Furthermore, an active equivalence principle applies in GRT, namely that different matter or radiation produce the same gravitation if only their energy-momentum tensor agrees.[1.]

\section{References:}

1. Lämmerzahl, Claus, Dittus, Hansjörg: Das Äquivalenzprinzip auf dem Prüfstand. In: Physik in unserer Zeit. 1999, Heft 2.

2. Will, Clifford M.: The confrontation between general relativity and experiment. Abschnitt 2.1. The Einstein Equivalence Principle. In: emis.de. Living Reviews in Relativity, 2014

3. Bessel F.W.: Versuche über die Kraft mit welcher die Erde Körper von verschiedener Beschaffenheit anzieht. Berlin 1832.

4. Roll,P.G., Krotkov,R., Dicke,R.H.: The equivalence of inertial and passive gravitational mass. In: Annals of Physics. 26 (1964), $442-$ 517, doi:10.1016/0003-4916(64)90259-3.

5. Shapiro,Iwin I., Counselman, Charles C. III, King, Robert W.: Verification of the Principle of Equivalence for Massive Bodies. In: Phys. Rev. Lett. 36 (1976), 555-558, doi:10.1103/PhysRevLett.36.555.

6. Rutherford Appleton Lab.: STEP: Satellite Test of the Equivalence Principle. (Memento from 16. Juli 2011 in Internet Archive). Zitat: „STEP aims to measure Equivalence at the level of 1 part in 1018."

7. Einstein, Albert: Über das Relativitätsprinzip und die aus demselben gezogenen Folgerungen Archiviert vom Original am 9.März 2017. In: Jahrbuch der Radioaktivität. 4, S.411-462.

8. Einstein, Albert: Lichtgeschwindigkeit und Statik des Gravitationsfeldes. In: Annalen der Physik. Bd. 38, 1912, S. 355, Online. (Memento vom 7. März 2016 im Internet Archive).

9. Einstein, Albert: Über den Einfluß der Schwerkraft auf die Ausbreitung des Lichtes. In: Annalen der Physik. Bd. 35, 1911, S. 898.

10. T Fließbach,T.: Allgemeine Relativitätstheorie. Spektrum (2006), ISBN 978-3-8274-1685-8, S. 51.

11. Dragon, Norbert: Geometrie der Relativitätstheorie. (Memento vom 19. April 2009 im Internet Archive). (PDF; 2,5MB).

12. Feynman, Richard: Surely You're Joking, Mr. Feynman!: Adventures of a Curious Character, Richard Feynman, Ralph Leighton (contributor), Edward Hutchings (editor), 1985, W. W. Norton, ISBN 0-393-01921-7, 1997 paperback: ISBN 0-393-31604-1, 2002 Blackstone Audiobooks unabridged audio cassette: ISBN 0-7861-2218-8 